\title{
Estimate of the Anesthesia Stage in Southern Elephant Seals using WEKA Data Mining Tool
}

\author{
M. D. Zárate \\ Centro para el Estudio de Sistemas Marinos, \\ Centro Nacional Patagónico, Consejo Nacional de \\ Investigaciones Científicas y Técnicas (CESIMAR- \\ CENPAT-CONICET) \\ Boulevard Brown 2915 (U9120ACD), Puerto \\ Madryn, Chubut, Argentina
}

\author{
M. N. Lewis \\ Centro para el Estudio de Sistemas Marinos, \\ Centro Nacional Patagónico, Consejo Nacional de \\ Investigaciones Científicas y Técnicas (CESIMAR- \\ CENPAT-CONICET) \\ Boulevard Brown 2915 (U9120ACD), Puerto \\ Madryn, Chubut, Argentina
}

\begin{abstract}
Prediction systems are techniques that build and study new forecasts through a branch of the artificial intelligence called Machine Learning. In this work it is estimate the time that remains anesthetized a southern elephant seal to which you have applied a combination of drugs (Zoletil $\left.{ }^{\circledR}\right)$, the fundamental objective of anesthesia is to avoid risky situations to researchers studying this species. To know these times, data mining techniques and classification algorithms are used, particularly algorithms it were compared J48, SMO, Random Tree, NB Tree y Naïve Bayes with data mining tool WEKA and a data set containing the records of 96 individuals undergoing anesthesia procedure. It is concluded that after tests (Random Tree) was the classification algorithm that best responded, with an accuracy of $98.79 \%$.
\end{abstract}

\section{Keywords}

Classification, Machine Learning, WEKA, Anesthesia, Mirounga leonina

\section{INTRODUCTION}

The Working Group, under the denomination GEMM@ (acronym for the Grupo de Estudio de Macroecología Marina) from CENPAT-CONICET dedicates its efforts to the development of studies on landscape ecology of marine species, using software tools and technological instruments for that purpose. In this context the population of Southern Elephant Seals (SES) is of great interest due to the fact that within populations of SES in the world, the colony of Peninsula Valdes (located in the Argentinean Sea, in the province of Chubut, Argentina) is the only one that presents a marked growth [1]. For the conservation and study of the species, it is required to obtain samples of blood, tissue, weight of the specimen, taking measures and make the placement of electronic equipment for the subsequent satellite tracking once they leave the coasts and migrate to the sea for several months. In particular, the deployment of miniature electronic devices in SES provides oceanographic profiles of the water column to depths of 2000 meters thus completing information gaps or strengthening the existing [2] [3]. These instruments returned, at a lower cost, large volumes of hydrographic data in regions that have never been studied directly by buoys or Research vessels this technique is known as biologging [4]

All these tasks require the immobilization of the SES, this it requires the use of anesthetic drugs. The procedure consists in injecting intramuscularly dose of Zoletil ${ }^{\circledR}$ (combination of
Tiletamine, a dissociative anesthetic agent and Zolazepam, a sedative, an anxiolytic and a muscle relaxant.). After the SES enters into what is known as induction stage, that is to say that is partially anesthetized proceeded to the placement of an intravenous injection with the same combination of drugs for this way to anesthetize completely the individual, this stage is known as anesthetic stage. Usually the second dose intravenously is performed with adult individuals due to the body volume of the same, while in young individuals enough with a dose of the drug intramuscular route to induce the anesthetic stage. It is at this time when you can start tasks of collecting samples of blood, tissue, and placement of electronic instruments. After a certain period of time the SES retrieves your state of consciousness and once assimilated fully the drug can return to its normal state. (See Figure 1)

Know the time of anesthesia is fundamental to researchers because accidents can occur if the SES try to defend himself, considering its average volume, the harm they can cause is a risk factor that must always be kept in mind.

In response to the need to establish the safe working times, it was necessary to go to the computer systems of automated learning with the objective of determine, using classification algorithms, the factors that contributed most to the estimation of safe working times.

The paper is organized as follows: Section 2 is a review of the techniques used in anesthesia animal, Section 3 describes the methodology adopted by us, and presents the anesthesia dataset used in this paper for analysis purpose; Section 4 describes data mining techniques used in this paper for analysis; result and conclusions are presented in Section 5 and Section 6 respectively.

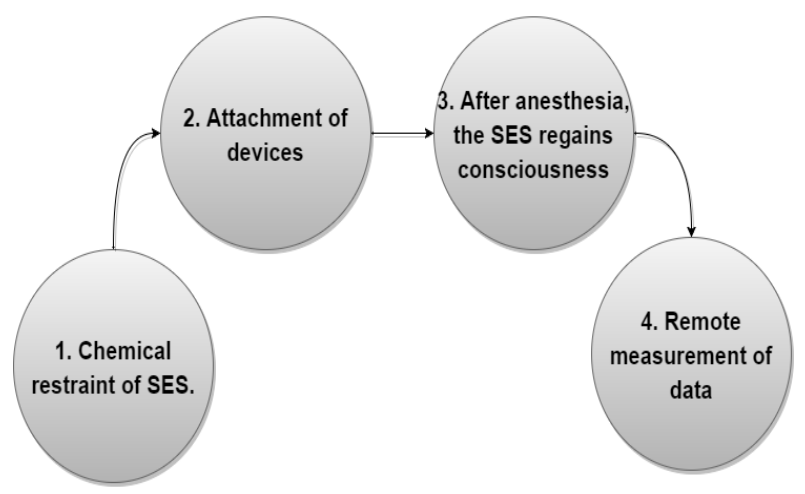

Fig. 1: Steps Performed during the Anesthesia Procedure 


\section{RELATED WORK}

To understand the different methods of immobilization and drugs that are commonly used with Mirounga leonina specie, were reviewed papers that are taken as reference in this area. First it was investigated the different methods of immobilization used in species of pinnipeds [5] then is taken as reference [6] [7] which describes the different combinations of drugs and their effects on SES and finally in [8] shows the effect that has the Zoletil ${ }^{\circledR}$ in elephant seals of different size, age, etc.

\section{METHODOLOGY}

To achieve the objective was proposed the following steps:

(i) Pre-processing of the available data of anesthesia.

(ii) The implementation of the different algorithms to estimate the time of anesthesia.

(iii) Comparison of the results obtained and selection of the most accurate algorithm.

The estimate and the prediction can be seen as types of classification. The general problem is to evaluate the model through the training data set and then check the result using a set of test data. For this study have used the tool WEKA (acronym of Waikato Environment for Knowledge Analysis), the same is born from the effort of a group of researchers from the Machine Learning Laboratory of the University of Waikato in New Zealand, as open source software under the terms of the GNU GPL which is a complete suite of tools for automatic programming and data mining [9] [10]. WEKA has four applications interfaces and each of them has different functions (For this paper the first two interfaces are used)

(i) Explorer: preprocessing, attribute selection, learning, visualization

(ii) Experimenter: testing and evaluating machine learning algorithms.

(iii) Knowledge Flow: Visual design for KDD process.

(iv) Simple CLI: a simple command interface without GUI.

Based on anesthesia procedures performed on SES in the Valdés Peninsula during the years 1998 to 2007, it has 96 records procedures anesthesia of different individuals, particularly these are the group "Weaning Pup" and for each instance it consider the following attributes: identifier (ID), Sex, Days Since weaning, Dose in mg. of Zoletil®, Effect of induction in minutes, Recovery time in minutes, Length in $\mathrm{Cm}$., Girth in $\mathrm{Cm}$. and weight calculated based on the length and girth [11]. Other attributes such as times and dates were removed because they are not relevant, also a new attribute (combination of another two) representing the relationship between the dose of Zoletil ${ }^{\circledR}$ supplied and weight of the individual it was added. The recovery times are shown in minutes and for the data set that is taken into consideration, this range is from 18 to 65 minutes, four time intervals were defined by assigning a nominal value as can be seen in Table 1.
Table 1. Time intervals for the Classification

\begin{tabular}{|c|c|}
\hline Recovery time & Interval in minutes \\
\hline FAST & $(-$ inf, 26$]$ \\
\hline NORMAL & $(26,34]$ \\
\hline SLOW & $(34,42]$ \\
\hline VERY SLOW & $(42,+$ inf $)$ \\
\hline
\end{tabular}

The data set used had 14 attributes (columns) of which were used for simplicity and to improve the estimation only those seen in Table 2.

Table 2. Attributes Selected after Pre-processing

\begin{tabular}{|c|c|}
\hline Name & Description \\
\hline Sex & Sex of the SES (M, F) (Nominal). \\
\hline Dose (mg.) & $\begin{array}{c}\text { Quantity of drugs injected in mg. } \\
\text { (Numeric). }\end{array}$ \\
\hline Weight (kg.) & $\begin{array}{c}\text { Weight calculated on the basis of the } \\
\text { length and the circumference of the SES } \\
\text { (Numeric). }\end{array}$ \\
\hline $\begin{array}{c}\text { Ratio (dose/ } \\
\text { weight). }\end{array}$ & $\begin{array}{c}\text { The quotient between the delivered dose } \\
\text { and the weight of the SES (Numeric). }\end{array}$ \\
\hline Rec. Time & $\begin{array}{c}\text { Time in which the SES is retrieved from } \\
\text { the effect of the drug (Nominal). }\end{array}$ \\
\hline
\end{tabular}

\section{DATA MINING TECHNIQUES}

The objective of this work is to compare different classification algorithms using WEKA to predict time of recovery, after the anesthetic drug is injected. In this case it has a file with data that contain 96 records of anesthesia in CSV format originally, but who were converted to the format ARFF (Attribute-Relation File Format) used by WEKA. The algorithms elected to perform the tests were J48 decision tree, SMO, Random Tree, NB tree and Naïve Bayes.

In all cases it was used the same set of data in the format arff, for this paper was used the file "anaesthetics.arff". For the tests were worked with the Explorer interface of WEKA, doing different tests with the classification algorithms. In all cases the option chosen was "Percentage Split" which by default is $66 \%$ (This means that will use the $66 \%$ of the data to train the algorithm and the rest will be for testing) then, will select the attribute to be classified, in this case Rec. Time (min). To perform comparatives of the selected algorithms, it was used the Experimenter interface, where it can see more detail each of the algorithms and choose the best result obtained. Before you begin to show the results of each of the algorithms it can see that in the Preprocess tab of the interface Explorer it is possible to see the attributes of the data set used, here its allows to delete attributes, apply filters, etc. Figure 2 shows this tab, in addition it can visualize the frequency distribution of all attributes at the same time by clicking on the button Visualize All. 


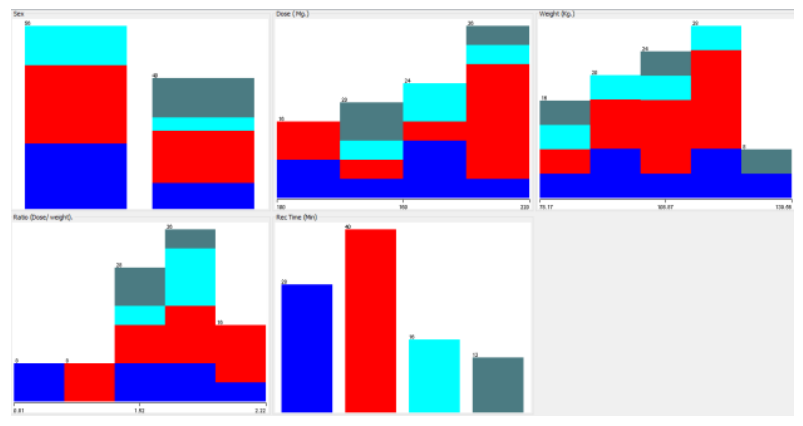

Fig. 2: Frequency distribution of all attributes in the dataset. Rec Time (min) have ranges from 18 to 65 minutes including 28 SES which recovered in the interval FAST (blue), 40 in the NORMAL range (red), 16 in the interval SLOW (Cyan) and 12 SES in the interval VERY SLOW (Gray)

Before you begin to evaluate the algorithms selected, was established the following performance metrics:

(i) Time: This is referred to as the time required to complete training or modeling of a dataset. It is represented in seconds

(ii) Kappa Statistic: A measure of the degree of nonrandom agreement between observers or measurements of the same categorical variable.

(iii) Mean Absolute Error: Mean absolute error is the average of the difference between predicted and the actual value in all test cases; it is the average prediction error.

(iv) Mean Squared Error: Mean-squared error is one of the most commonly used measures of success for numeric prediction. This value is computed by taking the average of the squared differences between each computed value and its corresponding correct value. The mean-squared error is simply the square root of the mean-squared-error. The meansquared error gives the error value the same dimensionality as the actual and predicted values.

(v) Root relative squared error: Relative squared error is the total squared error made relative to what the error would have been if the prediction had been the average of the absolute value. As with the root means-quared error, the square root of the relative squared error is taken to give it the same dimensions as the predicted value.

(vi) Relative Absolute Error: Relative Absolute Error is the total absolute error made relative to what the error would have been if the prediction simply had been the average of the actual values.

\subsection{J48 Decision Tree}

The algorithm J48 implemented in WEKA is a version of the classic Decision Trees algorithm C4.5 [12]. The Decision Trees fall within the methods of supervised classification, is to say that it has a dependent variable or class, and the objective of the classifier is to determine the value of that class for new cases. The algorithm J48 extends the functionality of the C4.5, such as allowing the realization of the process of post-pruning of the tree using a method based on the reduction of the error or that the divisions on the discrete variables are always binary. In the tab Classify is selected the algorithm J48 from among the trees of decision that gives us WEKA and proceed to the implementation. As can be seen after the completion of implementation, shows the output in text format, (see Table 3 ). The results obtained were the following: It can be seen that it is correctly classified the $90.9091 \%$ of instances and incorrectly the $9.0909 \%$.

Table 3. J48 Algorithm Accuracy

\begin{tabular}{|c|c|}
\hline Parameter & J48 \\
\hline Time taken to build model & $0.01 \mathrm{~s}$ \\
\hline Correctly Classified Instances & $90.90 \%$ \\
\hline Incorrectly Classified Instances & $9.09 \%$ \\
\hline Kappa statistic & 0.8576 \\
\hline Mean absolute error & 0.0417 \\
\hline Root mean squared error & 0.1872 \\
\hline Relative absolute error & $11.87 \%$ \\
\hline Root relative squared error & $45.61 \%$ \\
\hline
\end{tabular}

\subsection{Sequential Minimal Optimization}

Sequential Minimal Optimization (SMO) implements John C. Platt's sequential minimal optimization (SVM) [13] algorithm for training a support vector classifier using polynomial or RBF kernels. This implementation globally replaces all missing values and transforms nominal attributes into binary ones. It also normalizes all attributes by default. After running the algorithm the results obtained were the following: it is correctly classified the $42.4242 \%$ of instances and incorrectly the $57.5758 \%$ as can be seen in Table 4 .

Table 4. SMO Algorithm Accuracy

\begin{tabular}{|c|c|}
\hline Parameter & SMO \\
\hline Time taken to build model & $0.69 \mathrm{~s}$ \\
\hline Correctly Classified Instances & $42.42 \%$ \\
\hline Incorrectly Classified Instances & $57.57 \%$ \\
\hline Kappa statistic & 0.1157 \\
\hline Mean absolute error & 0.3207 \\
\hline Root mean squared error & 0.4098 \\
\hline Relative absolute error & $91.43 \%$ \\
\hline Root relative squared error & $99.8567 \%$ \\
\hline
\end{tabular}

\subsection{Random Tree}

Random Trees are essentially the combination of two existing algorithms in Machine Learning: single model trees are combined with Random Forest [14] ideas. Model trees are decision trees where every single leaf holds a linear model which is optimized for the local subspace described by this leaf. Random Forests have shown to improve the performance of single decision trees considerably: tree diversity is generated by two ways of randomization. First the training data is sampled with replacement for each single tree like in Bagging. Secondly, when growing a tree, instead of always 
computing the best possible split for each node only a random subset of all attributes is considered at every node, and the best split for that subset is computed. Such trees have been for classification Random model trees for the first time combine model trees and random forests. Random trees employ this produce for split selection and thus induce reasonably balanced trees where one global setting for the ridge value works across all leaves, thus simplifying the optimization procedure. The result obtained after execution of the algorithm, shown in Table 5, in this case is correctly classified the $100 \%$ of the instances, with a $0 \%$ of instances incorrectly classified.

Table 5. Random Tree Algorithm Accuracy

\begin{tabular}{|c|c|}
\hline Parameter & Random Tree \\
\hline Time taken to build model & $0.01 \mathrm{~s}$ \\
\hline Correctly Classified Instances & $100 \%$ \\
\hline Incorrectly Classified Instances & $0 \%$ \\
\hline Kappa statistic & 1 \\
\hline Mean absolute error & 0 \\
\hline Root mean squared error & 0 \\
\hline Relative absolute error & $0 \%$ \\
\hline Root relative squared error & $0 \%$ \\
\hline
\end{tabular}

\subsection{Naïve Bayes}

Naïve Bayes [15] is an intuitive method that uses the probabilities of each attribute belonging to each class to make a prediction. Naïve Bayes simplifies the calculation of probabilities by assuming that the probability of each attribute belonging to a given class value is independent of all other attributes. This is a strong assumption but results in a fast and effective method. The probability of a class value given a value of an attribute is called the conditional probability. By multiplying the conditional probabilities together for each attribute for a given class value, we have a probability of a data instance belonging to that class. To make a prediction, it can be calculate probabilities of the instance belonging to each class and select the class value with the highest probability. The result obtained after execution of the algorithm, shown in Table 6 , in this case is correctly classified the $48.4848 \%$ of the instances, with a $51.5152 \%$ of instances incorrectly classified.

Table 6. Naïve Bayes Algorithm Accuracy

\begin{tabular}{|c|c|}
\hline Parameter & Naïve Bayes \\
\hline Time taken to build model & $0 \mathrm{~s}$ \\
\hline Correctly Classified Instances & $48.48 \%$ \\
\hline Incorrectly Classified Instances & $51.51 \%$ \\
\hline Kappa statistic & 0.2208 \\
\hline Mean absolute error & 0.2887 \\
\hline Root mean squared error & 0.4015 \\
\hline Relative absolute error & $82.30 \%$ \\
\hline Root relative squared error & $97.82 \%$ \\
\hline
\end{tabular}

\subsection{NB Tree}

This algorithm [16] is basically a decision tree with Naïve Bayes classifiers in the leaves. The result obtained after execution of the algorithm, shown in Table 7, in this case is correctly classified the $93.9394 \%$ of the instances, with a $6.0606 \%$ of instances incorrectly classified.

Table 7. NB Tree Algorithm Accuracy

\begin{tabular}{|c|c|}
\hline Parameter & NB Tree \\
\hline Time taken to build model & $0.89 \mathrm{~s}$ \\
\hline Correctly Classified Instances & $93.93 \%$ \\
\hline Incorrectly Classified Instances & $6.06 \%$ \\
\hline Kappa statistic & 0.8966 \\
\hline Mean absolute error & 0.1306 \\
\hline Root mean squared error & 0.2129 \\
\hline Relative absolute error & $37.22 \%$ \\
\hline Root relative squared error & $51.88 \%$ \\
\hline
\end{tabular}

The results obtained through the training and the evaluation of the results showed a good performance in the classification of new times of recovery using the algorithm Random Tree.

\section{RESULTS}

While in the previous section it have a fairly accurate estimate about which algorithm presented better results, to be able to corroborate this, it will make use of the Experimenter interface of WEKA which allows us to know in detail the performance of each of the algorithms used, this process is to select the five algorithms that are used applied to data set anaesthetics.arff, each algorithm is run 10 times and used 66\% of the data set for training and the remaining 34\% for testing. Once you have configured the experimenter proceeded to run the tests from the tab run, the results of the tests are stored in an arff file and can be viewed from the Analyzes tab by opening the file that was created in Setup as can be seen in Figure 3 .

The correct percentage for each of the four algorithms is: 90.82 for J48, 98.79 for Random Tree, 47.27 For Naïve Bayes, 42.99 for SMO and 83.84 for NB Tree. The notation "v" or "*" indicates that a particular result is statistically better (v) or worse $(*)$ that the base algorithm (in this case, J48) at the level of significance specified (currently 0.05 )

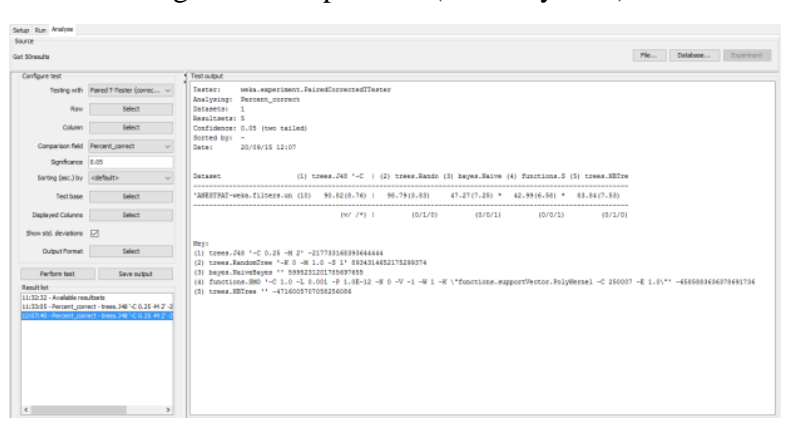

Fig. 3: Screenshot view of Experimenter Algorithm Accuracy

At the foot of each column you can see a legend (v//*) This is the number of times that the algorithm was better (v) equal ( ) 
or worse $(*)$ to the selected base algorithm (J48) taking into account the number of data sets that were used for the experiment (in this case is only used one). The results show that the Random Tree and NB Tree were equal $(0 / 1 / 0)$ that J48, while SMO and Naive Bayes were worse (0/0/1) that J48. Taking into account the previous results can be set that the best ranking algorithm of the five elected is the Random Tree due to its accuracy with respect to J48 $(90.82 \%)$ was $98.79 \%$.

\section{CONCLUSIONS}

The present work is used to estimate the time of anesthetic level then provided a dose of Zoletil ${ }^{\circledR}$ by intramuscular route to SES using WEKA data mining tool. In particular is used the data of the work carried out on the shores of the Valdés Peninsula with the category Weaning Pups. To achieve this objective is proposed to build a prediction model using techniques of classification ( $98.79 \%$ for Random Tree) in order to estimate new times of response to anesthesia provided on the basis of a number of attributes such as (sex, weight, dose/weight, etc.), this is of great help to the researchers working on the conservation of this species, because of the risk that exists when you are working with these individuals to take biological samples.

The future work will be focused on using the other classification algorithms of data mining. It is a known fact that the performance of an algorithm is dependent on the domain and the type of the data set. Hence, the usage of other classification algorithms like machine learning will be explored in future. In addition WEKA can use the library to build an expert system that allows establish safe working times using a mobile application designed specifically for this purpose.

\section{REFERENCES}

[1] Lewis, M. Campagna C. y F. Quintana. 1996. Site fidelity and dispersion of southern elephant seals from patagonia. Marine Mammal Science 12(1):138-147.

[2] Boehme, L., P. Lovell, M. Biuw, F. Roquet, J. Nicholson, S. E. Thorpe, M. P. Meredith, and M. Fedak, 2009: Technical note: Animal- borne CTD-satellite relay data loggers for real-time oceanographic data collection. Ocean Sci., 5, 685-695.

[3] Roquet, F. et al. Estimates of the southern ocean general circulation improved by animal-borne instruments. Geophys. Res. Lett. 40, 6176-6180 (2013).

[4] Rutz, C., \& Hays, G. C. (2009). New frontiers in biologging science. Biology letters, 5(3), 289-292.
[5] Gales NJ. Chemical restraint and anaesthesia of pinnipeds: a review. Mar Mamm Sci 1989; 5:228-256.5.

[6] Woods R, McClean S, Nicol S, Burton H. A comparison of some cyclohexamine based drug combinations for chemical restraint of southern elephant seals (Mirounga leonina). Vet Rec. 1994; 10:412-429.

[7] Woods R, McClean S, Nicol S, Burton H. Antagonism of some cyclohex-amine-based drug combinations used for chemical restraint of southern elephant seals (Mirounga leonina). Aust Vet J. 1995; 72:165-17.

[8] McMahon, C. R., Burton, H., McLean, S., Slip, D., \& Bester, M. (2000). Field immobilization of southern elephant seals with intravenous tiletamine and zolazepam. Veterinary Record, 146(9), 251-254.

[9] Garner, S. R. (1995, April). Weka: The waikato environment for knowledge analysis. In Proceedings of the New Zealand computer science research students conference (pp. 57-64).

[10] Hall, M., Frank, E., Holmes, G., Pfahringer, B., Reutemann, P., \& Witten, I. H. (2009). The WEKA data mining software: an update. ACM SIGKDD explorations newsletter, 11(1), 10-18.

[11] Haley, M. P., Deutsch, C. J., \& Boeuf, B. J. L. (1991). A method for estimating mass of large pinnipeds. Marine Mammal Science, 7(2), 157-164.

[12] Quinlan, J. R. (2014). C4. 5: programs for machine learning. Elsevier.

[13] J. Platt: Fast Training of Support Vector Machines using Sequential Minimal Optimization. In B. Schoelkopf and C. Burges and A. Smola, editors, Advances in Kernel Methods - Support Vector Learning, 1998.

[14] Breiman, L. (2001). Random forests. Machine learning, 45(1), 5-32.

[15] John, G. H., \& Langley, P. (1995, August). Estimating continuous distributions in Bayesian classifiers. In Proceedings of the Eleventh conference on Uncertainty in artificial intelligence (pp. 338-345). Morgan Kaufmann Publishers Inc.

[16] Kohavi, R. (1996, August). Scaling Up the Accuracy of Naive-Bayes Classifiers: A Decision-Tree Hybrid. In KDD (pp. 202-207). 